\title{
CARDIOTOXICIDAD POSTERIOR A LA INTOXICACIÓN POR AMITRAZ. REPORTE DE CASO Y REVISIÓN DE LA LITERATURA
}

\author{
AbNer Lozano L. MD. Internista ${ }^{1}$ y Oswaldo JaVier Tovar P. MD. Toxicólogo ${ }^{2 *}$ \\ ${ }^{1}$ Especialista en Medicina Crítica y Cuidados Intensivos, coordinador UCI adultos Hospital Universitario \\ Hernando Moncaleano Perdomo, Profesor Asociado de la Universidad Surcolombiana, Neiva, Huila, Colombia. ${ }^{2}$ \\ Secretaria de Salud Departamental del Huila. Residente III año de Medicina Interna, Universidad Surcolombiana. \\ Servicio de Urgencias Hospital Universitario Hernando Moncaleano Perdomo; Neiva, Huila, Colombia.
}

\begin{abstract}
Resumen
La intoxicación por amitráz típicamente genera una variedad de síntomas tales como bradicardia e hipotensión, con colapso hemodinámico, que pueden ocasionar un riesgo para la vida; además se presenta depresión neurológica y respiratoria, todos estos explicados por una caída del tono simpático a nivel central y periférico. Los defectos en el ritmo cardiaco no son la característica mas sobresaliente de este tipo de intoxicación puesto que no se han descrito efectos cardiotóxicos directos ocasionados por este agente químico. En este artículo se reporta un caso en donde se observa defecto de la conducción cardiaca posterior a la ingestión de amitráz. La toxicidad cardiovascular se manifestó con hipotensión, bradicardia, y además incluyó un bloqueo aurículo-ventricular de II grado Mobitz I, que se resolvió espontáneamente después de 24 de monitorización.
\end{abstract}

Palabras clave: amitráz, intoxicación, bloqueo aurículoventricular

\section{CARDIOTOXICITY FOLLOWING AMITRAZ POISONING: A CASE REPORT AND LITERATURE REVIEW}

\begin{abstract}
Amitraz poisoning typically produces a variety of symptoms, among others bradycardia and hypotension with hemodynamic collapse, that can be life-threatening; it can also show neurological and respiratory depression, all these explained by a fall in the sympathetic tone at central and peripheral level. Defects in heart rate are not the most outstanding characteristic of this sort of intoxication, since no direct cardio toxic effects have been proven to be caused by this chemical. This article reports a case where cardiac conduction defect is observed after ingestion of amitraz. Cardiovascular toxicity was manifested as hypotension, bradycardia, and also included was a second degree atrioventricular Mobitz I block, which spontaneously reverted after a 24 hour monitoring.
\end{abstract}

Key words: amitraz, poisoning, atrioventricular block

* Correspondencia: Oswaldo J. Tovar osjatopu@hotmail.com. Dirección postal: Carrera 8 No 204F-25, Universidad Surcolombiana, Neiva, Huila, Colombia.

Recibido: Marzo 18 de 2010 Aceptado: Diciembre 1 de 2010 


\title{
CARDIOTOXICIDADE APÓS INTOXICAÇÃO PELO AMITRAZ. RELATÓRIO DE CASO E REVISÃO DA LITERATURA
}

\begin{abstract}
Resumo
A intoxicação por amitraz tipicamente produz uma variedade de sintomas como bradicardia e hipotensão arterial, com colapso hemodinâmico, que pode causar risco de vida; também apresentam depressão neurológica e respiratória, todos esses explicado por uma queda do tônus simpático, a nível central e periférico. Os defeitos da freqüência cardíaca não são a característica mais marcante de intoxicação, porém não tem sido descritos efeitos cardiotóxicos diretos causados por esta substância. Este artigo relata um caso que mostra um defeito de condução cardíaca após a ingestão de amitraz. A toxicidade cardiovascular foi manifestada como hipotensão, bradicardia, e também incluiu um bloqueio atrioventricular de segundo grau Mobitz I, que resolveu-se espontâneamente após 24 de monitorização.
\end{abstract}

Palavras-chave: amitraz, intoxicação, bloqueio atrioventricular

\section{Introducción}

El amitráz es un pesticida formamidínico (diaminidas) ampliamente utilizado para el control de insectos y parásitos (ácaros y garrapaticida) tanto en la agricultura como en la medicina veterinaria, de venta libre en nuestro país y se consigue bajo diferentes nombres comerciales (Garratraz ${ }^{\circledR}$, Taktic ${ }^{\circledR}$, Ivomec ${ }^{\circledR}$ Triatox ${ }^{\circledR}$, Ectodex ${ }^{\circledR}$, Mitac $®$, Ganabaño $\left.{ }^{\circledR}\right)(1)$. En Colombia, son pocos los casos de intoxicación reportados y en la literatura médica mundial, la mayor parte de los reportes son asociados a la exposición accidental y han sido raros los casos relacionados con la ingesta voluntaria con fines suicidas $(1,2)$.

Los efectos toxicológicos del amitráz son debidos a la estimulación de los receptores alfa-2 a nivel central y periférico, que ocasiona una caída del tono adrenérgico. También inhibe la síntesis de prostaglandinas a nivel hipotalámico y la actividad enzimática de la monoamino oxidasa (MAO) a nivel central y periférico $(1,3)$. La toxicidad reportada en los animales y en los humanos afecta varios sistemas, entre estos al sistema nervioso central causando depresión neurológica y respiratoria, convulsiones, miosis o midriasis, hipo o arreflexia, hipotermia; al sistema cardiovascular produciendo hipotensión y bradicardia, que pueden ocasionar la muerte si no se revierten en forma oportuna; al sistema gastrointestinal causando nausea, vómito, îleo intestinal, distensión abdominal y al sistema endocrino ocasionando hiperglucemia y poliuria. Se observa también, que gran parte de la sintomatología clínica de la intoxicación es causada principalmente por la combinación del amitráz y su vehículo, el xileno (1-3).
La mayoría de los casos de intoxicación por amitráz son manejados en las salas generales de los servicios de urgencias; sin embargo, los casos más severos de envenenamiento deben ser soportados con ventilación mecánica y con agentes inotrópicos/vasopresores en las Unidades de Cuidados Intensivos por breves periodos (50 \pm 16 horas), y los pacientes deben permanecer hospitalizados por periodos variables de tiempo (5-11 días). La morbimortalidad es bastante baja en este grupo de pacientes y siendo las complicaciones infecciosas, cardiovasculares, neurológicas y electrolíticas las mayores causas de hospitalización y de muerte (1).

Tanto en niños y como en adultos intoxicados por amitráz se han reportado trastornos de la repolarización intraventricular en el electrocardiograma, los cuales son transitorios y revierten en forma espontánea (4). Sin embargo, no se han descrito efectos cardiotóxicos directos relacionados con el amitráz que afecten el sistema de conducción, tanto a nivel de los canales iónicos o el sistema de bombas que intervienen en el ciclo cardiaco.

\section{Intoxicación por Amitráz}

La intoxicacion por amitráz en los humanos puede ocurrir por diferentes vías como la oral, la dérmica o por inhalación; la ingestión de esta sustancia es la forma más frecuente utilizada con fines suicidas. Las preparaciones comerciales del amitráz contienen entre $12,5-20 \%$ del compuesto principal (principio activo) disuelto en solventes orgánicos tales como el xileno; cuando va a ser utilizado para fumigar cosechas $y$ para desparasitar el ganado, se diluye en agua unas 
100-600 veces $(1,2)$. La dosis tóxica del amitráz está entre 89,2 y $163 \mathrm{mg} / \mathrm{kg}(5,6)$.

Los mecanismos de toxicidad del amitráz y los efectos sobre los diferentes sistemas y órganos son:

1. Nervioso: sedación y depresión neurológica debida a la estimulación de los receptores alfa 2 centrales $(3,4,8,9)$. Además el Xileno puede causar toxicidad aguda a nivel del sistema nervioso central manifestada por depresión neurológica, que puede oscilar entre somnolencia, estupor hasta el coma y depresión respiratoria, ataxia, incoordinación, nistagmus, convulsiones y neuroirritabilidad $(1,2)$.

2. Respiratorio: efecto depresor directo sobre el centro respiratorio con inhibición de la respuesta al $\mathrm{CO}_{2}(5)$.

3. Cardiovascular: agonismo sobre los receptores alfa-2 periféricos, vasoplejia por estimulación de la síntesis y la liberación del factor relajante del endotelio (óxido nítrico) e inhibición de la liberación pre-sináptica de noradrenalina y aminas simpaticomiméticas causando hipotensión y bradicardia $(5,7)$.

4. Termoregulación: efecto directo sobre el centro regulador hipotalámico (inhibiendo la síntesis de prostaglandinas) lo que ocasiona hipotermia. La hipotermia inducida por el amitráz es revertida por el potente antagonista del receptor alfa-2 (atimepazole) (6).

5. Pupila: miosis por estimulación pre-sináptica de los receptores alfa-2 (bajas dosis) y midriasis por estimulación post-sináptica de los receptores alfa-2 (altas dosis) $(5,7)$.

6. Renal: inhibición en la liberación a nivel central de la $\mathrm{ADH}$ y la activación del eje renina-angiotensinaaldosterona, lo que ocasiona aumento en la tasa de filtración glomerular y poliuria (7).

7. Gastrointestinal: inhibición en la liberación presináptica de acetilcolina a nivel de la terminación nerviosa parasimpática y efecto directo sobre receptores alfa-2 digestivos, lo que causa íleo y distensión abdominal (disminución del peristaltismo) $(8,9)$.

8. Endocrino: hiperglucemia, al inhibir la liberación pancreática de insulina y estimular la liberación de glucagón. Mecanismo mediado a través de su metabolito activo, el BTS $27271(9,10)$.

9. Hepático: elevación de las transaminasas hepáticas por inhibición en la actividad de la glutatión transferasa e igualmente por efectos hepatotóxicos directos ocasionados por los solventes orgánicos aromáticos (xileno) $(11,12)$.

Las manifestaciones clínicas de la intoxicación por amitráz pueden ser confundidas con la exposición a sustancias inhibidoras de la colinesterasa (organofosforados y carbamatos), lo mismo que la sobredosis con otros agentes de acción central como la clonidina; por lo tanto, se requiere un alto índice de sospecha ante la presencia de estos hallazgos clínicos y el antecedente de exposición a sustancias tipo insecticidas o ectoparasiticidas, lo mismo que diferenciarla de la intoxicación por sustancias depresoras del sistema nervioso central como opiodes, benzodiacepinas o barbitúricos, fenotiazinas y antidepresivos triciclicos $(2,5,12)$. El manejo inicial en los servicios de urgencias consiste en una completa historia clínica y examen físico, lo mismo que la toma de gases arteriales con electrolitos, electrocardiograma de doce derivaciones, glucemia central y pruebas de función hepática.

Una vez estabilizado el paciente, se puede considerar la realización del lavado gástrico, si la ingestión ocurrió dentro de la primera hora de consulta; sin embargo, la presencia de solventes hidrocarbonados es una contraindicación relativa para la realización de dicho procedimiento por el riesgo de broncoaspiración. Igualmente se debe iniciar la administración de carbón activado (50 gr) por vía oral o sonda nasogástrica; no hay evidencia médica de que las medidas de descontaminación gastrointestinal en la intoxicación por amitráz sean de beneficio clínico (13).

El manejo específico de los pacientes intoxicados por amitráz es de soporte y está dirigido a las principales manifestaciones clínicas de la intoxicación. Optimizar el manejo de la vía aérea, suministrando oxígeno a alto flujo y asegurarla en casos de depresión neurológica severa, la reanimación con soluciones cristaloides e inicio de sustancias vasopresoras (dopamina/noradrenalina) se recomiendan en los casos de hipotensión que no responde a la infusión de líquidos endovenosos. La atropina puede ser útil cuando se presenta bradiarritmia severa que causa inestabilidad hemodinámica y caída del gasto cardiaco (13). La mayor parte de revisiones y reportes de casos coinciden en que la recuperación del estado de conciencia varía entre $2-48$ horas $(14,15)$. En caso de convulsiones de deben utilizar la benzodiacepinas tipo diazepam o midazolam (16). La ventilación mecánica es una medida salvadora, en casos de depresión respiratoria severa (17). 
En la actualidad no existe un antídoto específico para tratar a los humanos intoxicados por amitráz. Sin embargo, experimentalmente en modelos animales se ha observado una completa reversión de los efectos clínicos y paraclínicos del envenenamiento cuando se han utilizado antagonistas del receptor alfa-2, como la yohimbina y el atimepazole, los cuales deben ser utilizados solamente en casos severos de intoxicación $(6,13,18)$. Aunque no se ha demostrado la utilidad de la naloxona, ha sido usada experimentalmente para revertir los efectos depresores respiratorios en algunos reportes de casos de intoxicación por amitráz $(19,20)$.

Los principales mecanismos de cardiotoxicidad son debidos a alteraciones en la electrofisiología de la conducción cardiaca, específicamente alterando el influjo o el eflujo de iones por bloqueo de canales eléctricos que intervienen en los procesos de despolarización y repolarización del músculo cardiaco (21). Existen diferentes blancos moleculares de las sustancias tóxicas dentro del sistema de conducción cardiaca, y son los responsables de sus efectos cardiotóxicos; entre ellos están:

- Bloqueo de los canales de sodio (fase 0 del potencial de acción del ciclo cardiaco) (21)

- Bloqueo de los canales de calcio (fase 1 del potencial de acción del ciclo cardiaco) (21)

- Bloqueo de los canales de potasio (fase 2-3 del potencial de acción del ciclo cardiaco) (21)

- Inhibición de la bomba sodio-potasio ATPasa (fase 4 del potencial de acción del ciclo cardiaco) (21)

- Bloqueo mixto de los canales de sodio, potasio y calcio (21)

- Inhibición de los receptores Beta adrenérgicos (21)

En este artículo se reporta un único caso de defecto de la conducción cardiaca posterior a la ingestión de amitráz. La toxicidad cardiovascular se manifestó con hipotensión, bradicardia, y además incluyó un bloqueo aurículo-ventricular de II grado Mobitz I, el cual resolvió espontáneamente después de 24 horas de monitorización.

\section{Reporte de caso}

Paciente de 28 años, natural de Buga, Valle del Cauca, procedente de la vereda Las Palmas, Algeciras (Huila), consulta al servicio de urgencias por haber ingerido voluntariamente un garrapaticida líquido (amitráz) una hora antes de ingresar al centro de salud; en el momento de la admisión presentaba compromiso del estado de conciencia en forma progresiva hasta el estupor profundo, miosis, deterioro del patrón respiratorio, tendencia a la hipotensión y bradicardia, presentando falla respiratoria, requiriendo intubación orotraqueal, soporte ventilatorio con AMBU y la administración endovenosa de $4 \mathrm{mg}$ de atropina, motivo por el cual se remite a un nivel de mayor complejidad. Los acompañantes referían disfunción conyugal con su actual pareja desde hace varios meses, con sentimientos de desesperación y desesperanza, sin una clara ideación suicida. Negaban antecedentes personales de importancia, con antecedentes ginecobstétricos: G2 P2 A0, último parto hace un año y planificación con perlutal intramuscular mensual.

Al ingreso a III nivel se encuentra intubada selectivamente, con alteración del estado de conciencia, estuporosa profunda, sin respiraciones espontáneas, distensión abdominal, ausencia de peristaltismo, no fasciculaciones en las extremidades, moviliza simétricamente al estímulo doloroso, discoria con anisocoria izquierda no reactiva y pupila derecha miótica poco reactiva de $3 \times 3 \mathrm{~mm}$. Se cambia tubo endotraqueal y se fija a $21 \mathrm{~cm}$ a nivel de la comisura labial, continua soporte respiratorio mediante ventilación con presión positiva con AMBU, se inicia sedación con midazolam a $3 \mathrm{mg} / \mathrm{h}$, se pasa catéter central de inserción periférica, además se inician soluciones cristaloides, profilaxis gástrica y anti-trombótica, monitoreo continuo, paso de sonda nasogástrica con salida de olor a hidrocarburo, paso de sonda vesical y se solicita admisión a UCI.

Durante la monitorización continua se evidencia tendencia a la hipotensión y bradicardia, requiriendo administración de $2 \mathrm{mg}$ de atropina. Durante la noche se decide retirar sedación por estabilidad hemodinámica con extubación programada con adecuada tolerancia de esta. Sin embargo, durante el transcurso del día siguiente presenta episodios de hipotensión y bradicardia con compromiso hemodinámico requiriendo reanimación con 2 litros de cristaloides y la administración de dopamina a $10 \mathrm{mg} / \mathrm{kg} / \mathrm{min}$, con estabilización de sus signos vitales.

Se solicita EKG de doce derivaciones donde se evidencia bloqueo aurículoventricular de II grado Mobitz I, con QTc normal, motivo por el cual se sospecha cardiotoxicidad $v$ s miocarditis de origen tóxico y se solicita ecocardiograma transtorácico, monitoreo electrocardiográfico continuo (Figuras 1-4). 


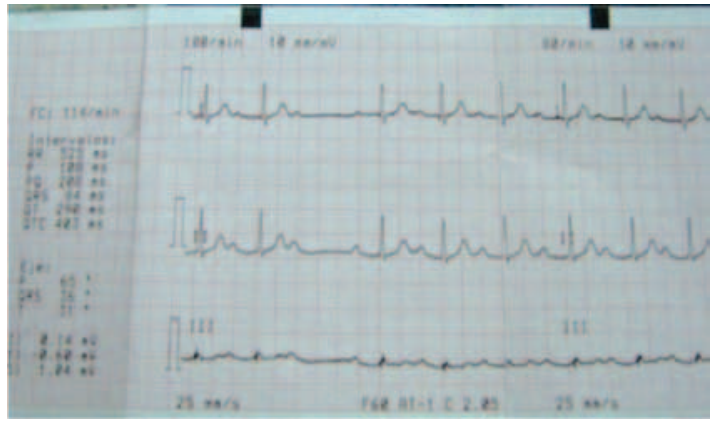

Figura 1. Electrocardiograma de superficie donde se observa que el intervalo PR se prolonga en forma progresiva hasta que ocurre el bloqueo.

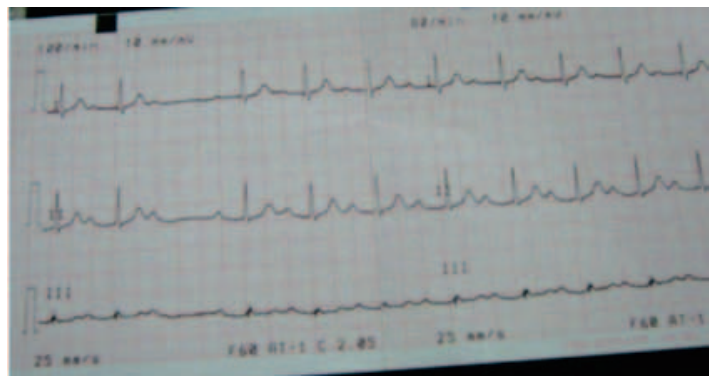

Figura 2. Electrocardiograma de superficie que muestra el bloqueo aurículoventricular de II grado Mobitz I.

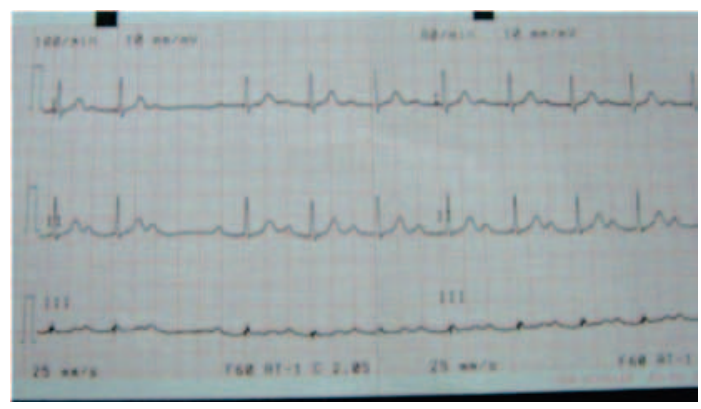

Figura 3. Electrocardiograma de superficie donde se observa bloqueo aurículoventricular de II grado Mobitz I. Se observa onda $P$ sola que no conduce.

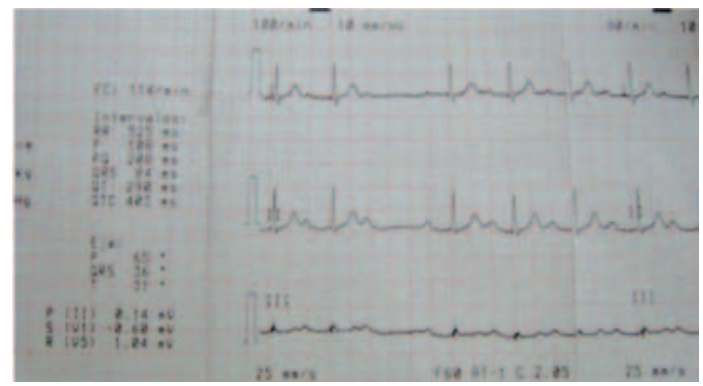

Figura 4. Electrocardiograma de superficie muestra Bloqueo aurículoventricular de II grado Mobitz I. Se observa QTC normal (403 mseg).
El estudio ecocardiográfico es interpretado como normal, con fracción de eyección del ventrículo izquierdo del $64 \%$, sin transtornos difusos o segmentarios de la contractilidad. La evolución clínica es adecuada con mejoría del estado neurológico, presencia de reflejos protectores de vía aérea, retirándosele la sonda nasogástrica y vesical, destete progresivo de soporte vasoactivo con dopamina. El nuevo electrocardiograma, tomado 24 horas después muestra solo taquicardia sinusal con resolución completa del bloqueo (Figura 5).

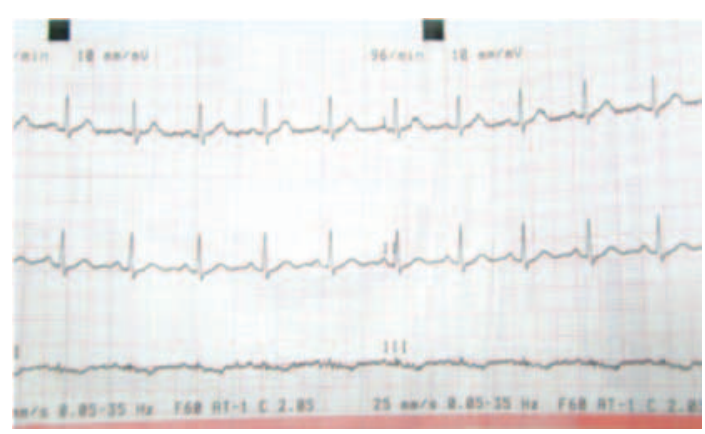

Figura 5. Resolución completa del Bloqueo aurículoventricular de II grado Mobitz I.

Se reinterroga nuevamente la paciente y manifiesta trauma penetrante en ojo derecho previo, pero sin compromiso de la agudeza visual. Al examen pupilar presenta discoria izquierda reactiva de $4 \times 4 \mathrm{~mm}$ y derecha de $4 \times 4 \mathrm{~mm}$ también reactiva, no hay compromiso oculomotor, ni de pares bajos. Durante la evolución clínica intrahospitalaria, presenta episodio febril; sin embargo, persiste con estabilidad hemodinámica, mejoría neurológica, con adecuado patrón respiratorio, y tolera destete de dopamina. Se documenta neumonía aspirativa basal derecha con derrame pleural paraneumónico, iniciándosele manejo con ampicilina sulbactam 1,5 gr IV cada seis horas por siete días, con adecuada evolución clínica (Figuras 6 y 7). Se da el alta para continuar control ambulatorio con psiquiatría y trabajo social.

Tabla No 1. Valores de los paraclínicos

\begin{tabular}{lcccc}
\hline & $\mathbf{2 8 - 1 0 - 0 9}$ & $\mathbf{3 0 - 1 0 - 0 9}$ & & $\mathbf{3 0 - 1 0 - 0 9}$ \\
\hline Leucocitos/mm3 & 7700 & 10.000 & $\begin{array}{c}\text { TGO } \\
\text { U/L }\end{array}$ & 32 \\
\hline Neutrofilos/mm 3 & 6000 & 8500 & $\begin{array}{c}\text { TGP } \\
\text { U/L }\end{array}$ & 22 \\
\hline Linfocitos/mm3 & 1400 & 900 & $\begin{array}{c}\mathrm{BT} \\
\mathrm{mg} \%\end{array}$ & 1,6 \\
\hline
\end{tabular}




\begin{tabular}{|c|c|c|c|c|}
\hline $\mathrm{Hb} / \mathrm{Hto}$ & $15,3 / 45$ & $13 / 38,8$ & $\begin{array}{c}\mathrm{BD} \\
\mathrm{mg} \%\end{array}$ & 0,3 \\
\hline Plaquetas/mm3 & 220000 & 191000 & $\begin{array}{c}\mathrm{BI} \\
\mathrm{mg} \%\end{array}$ & 1,3 \\
\hline Citoq de orina & cetonuria & & $\begin{array}{l}\mathrm{PCR} \\
\mathrm{mg} \% \\
\end{array}$ & 7,9 \\
\hline $\begin{array}{l}\text { Colinesterasa } \\
\text { plasmática } \\
\text { Normal: 4970- } \\
13997 \text { U/L. }\end{array}$ & $10780 \mathrm{U} / \mathrm{L}$ & & & \\
\hline Gravindez & Negativo & & & \\
\hline Gases arteriales & $\begin{array}{l}\text { Alcalosis } \\
\text { respiratoria } \\
\text { AGAP } 18\end{array}$ & & & $\begin{array}{c}\text { pH:7,4, Pco2 } \\
\text { 27,8, Po2 } \\
\text { 83,3, } \mathrm{HCO}_{3}: \\
\text { 17,4AGap:23 }\end{array}$ \\
\hline $\mathrm{Na}^{+} \mathrm{mmol} / \mathrm{l}$ & 148 & & & 141 \\
\hline $\mathrm{K}^{+} \mathrm{mmol} / \mathrm{L}$ & 3 & & & 3,8 \\
\hline $\mathrm{Cl}^{-m m o L} / \mathrm{l}$ & 109 & & & 105 \\
\hline
\end{tabular}

\section{Discusión}

El amitraz y su solvente xileno son utilizados en todo el mundo por sus propiedades insecticidas y ectoparasitidas. Pueden causar toxicidad en animales y en humanos al ser ingeridos en forma accidental o voluntaria, inhalada o al contacto con la piel. Muchos de los efectos toxicológicos del amitráz reportados en los humanos afectan varios sistemas e inclusive el solvente utilizado en los productos de amitráz como lo es el xileno, pueden causar toxicidad aguda a nivel del sistema nervioso central y cardiovascular $(1,2)$.

\section{Cardiotoxicidad por amitráz}

Se han reportado cambios inespecíficos del segmento ST en el electrocardiograma en niños y adultos intoxicados por amitráz, y que no presentaban antecedentes de enfermedad cardiaca previa, los cuales se revierten de forma espontánea dentro de las primeras 24 horas del envenenamiento (21). Sin embargo, no se han descrito efectos cardiotóxicos directos relacionados con el amitráz que afecten el sistema de conducción, canales iónicos o el sistema de bombas que intervienen en el ciclo cardiaco. Basados en los resultados observados, se puede pensar que la intoxicación por amitráz muy posiblemente originó la alteración del ritmo cardiaco que fue evidente dentro de las primeras 24 horas de evolución y que se resolvió en forma espontánea una vez se soportó la paciente con dopamina, aunque no se puede descartar que haya sido el xileno el responsable de la alteración del ritmo cardiaco.

Figura 6. Rx de tórax. Se observa infiltrados alveolares intersticiales basales derechos con consolidación y derrame pleural.

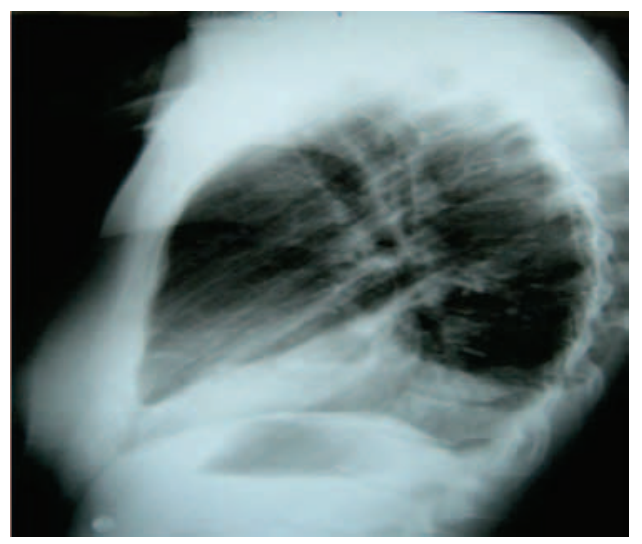

Figura 7. Rx de tórax. Se observa derrame pleural derecho.

En los pacientes intoxicados por amitráz generalmente se observa que las concentraciones de creatinina, nitrógeno ureico, sodio y potasio no se modifican; sin embargo, en algunos reportes de casos publicados se han informado casos de hiponatremia y elevación de transaminasas, situación que no fue observada en el caso presentado en este artículo. La alteración gasométrica probablemente esté relacionada con hipovolemia o hipoperfusión tisular por la inestabilidad cardiovascular y citogénesis por ayuno. Se descartó intoxicación por inhibidores de colinesterasa por la actividad normal de la enzima. Al ingreso de la paciente se detectó alteración hidroelectrolítica (hipokalemia), que si bien puede prolongar el tiempo de repolarización ventricular, este no se alteró, puesto que el intervalo QTc estaba dentro de rango normal (403 mseg). 
Una de las principales causas de morbimortalidad en este grupo de pacientes intoxicados son las complicaciones infecciosas; la paciente de este caso presentó una neumonía probablemente aspirativa que se resolvió adecuadamente con el tratamiento antimicrobiano instaurado.

Se debe tener en cuenta que hay muchos agentes químicos y tóxicos que causan alteraciones en el ciclo cardíaco, con los subsiguientes cambios electrocardiográficos que pueden manifestarse como bradiarritmias o taquiarritmias; sin embargo, hasta el momento no se han diseñado estudios clínicos para evaluar las alteraciones que estos agentes pueden causar, $e$ igualmente existen muy pocos reportes de casos en la literatura sobre este tipo de intoxicaciones (22). Por lo tanto se recomienda a los médicos que atienden a este grupo de pacientes que estén siempre atentos y vigilantes de cualquier cambio en el ritmo cardiaco que pueda potencialmente ocurrir en este grupo de pacientes intoxicados, que se hagan los reportes con un detallada descripción de los cambios observados, y de esa manera habrá mayor evidencia que permita evaluar las alteraciones causadas por esos agentes químicos. De igual forma el diagnóstico diferencial es importante para identificar las intoxicaciones por amitráz de las producidas por compuestos inhibidores de colinesterasas (organofosforados y carbamatos) que presentan síntomas y signos muy similares y así poder suministrar un tratamiento apropiado.

\section{Agradecimientos}

Al Dr. Jaime Molina por la traducción y preparación del manuscrito y a la secretaría de Salud Departamental del Huila por el apoyo logístico y recursos administrativos.

\section{Referencias}

1. Levent A. Acute Amitraz Poisoning in Adults: Clinical Features, Laboratory Findings, and Management. Clinical Toxicology. 2006;44:19-23.

2. Yilmaz H L, Yildizdas D R. Amitraz Poisoning, An Emerging Problem: Epidemiology, Clinical Features, Management, and Preventive Strategies. Arch Dis Child. 2003;88(2):130-134.

3. Jorens P G, Zandijk E, Belmans L, Schepens P J, Bossaert L L. An Unusual Poisoning with the Unusual Pesticide Amitraz. Human Exp Toxicol. 1997;16(10):600-1.
4. Aydin K, Kurtoglu S, Poyrazoglu M H, Uzum K, Ustunbas H B, Hallac I K. Amitraz Poisoning in Children: Clinical and Laboratory Findings of Eight Cases. Human Exp Toxicol. 1997;16(11):680-2.

5. Maze M, Tranquilli W. Alpha-2 Adrenoceptor Agonists: Defining the Role in Clinical Anaesthesia. Anaesthesiology. 1991;74(3):581-605.

6. Barkin R M, ed: Appendix A-2. Vital Sings and ancillary ventilator support: pediatric Emerg. Med. Conceps and clinical practice. St Louis: Mosby; 1997. p. 1117.

7. Jones R D. Xylene/Amitraz: A Pharmacologic Review and Profile. Vet Hum Toxicol. 1990;32(5):446-8.

8. Ulukaya S, Demirag K, Moral A R. Acute Amitraz Intoxication in Human. Intensive Care Med. 2001;27(5):930-3.

9. Hugnet C, Buronrosse F, Pineau X, Cadore J L, Lorgue G, Berny P J. Toxicity and Kinetics of Amitraz in Dogs. Am J Vet Res. 1996;57(10):1506-10.

10. Chemical Safety Information from Intergovernmental Organizations. Xylene. (Accessed March 26, 2005)

11. Abu-Basha E A, Yibchok-Anun S, Hopper D L, Hsu W H. Effects of the Pesticide Amitraz and its Metabolite BTS 27271 on Insulin and Glucagon Secretion from the Perfused Rat Pancreas: Involvement of alpha 2-adrenergic Receptors. Metabolism. 1999;48(11):1461-69.

12. Smith B E, Hsu W H, Yang P C. Amitraz-induced Glucose Intolerance in Rats: Antagonism by Yohimbine but not by Prazosin. Arch Toxicol. 1990;64(8):680-3.

13. Aydin K, Per H, Kurtoglu S, Poyrazoglu M H, Narin N, Aslan D. Amitraz Poisoning in Children. Eur J Pediatr. 2002;161(6):34950.

14. Ertekin V, Alp H, Selimoglu M A, Karacan M. Amitraz Poisoning in Children: Retrospective Analysis of 21 Cases. J Int Med Res. 2002;30(2):203-5.

15. Proudfoot A T. Poisoning with Amitraz. Toxicol Rev. 2003;22(2):71-4.

16. Atabek M E, Aydin K, Erkul I. Different Clinical Features of Amitraz Poisoning in Children. Human Exp Toxicol. 2002;21(1):13-6.

17. Doganay Z, Aygun D, Altintop L, Guven H, Bildik F. Basic Toxicological Approach has Been Effective in Two Poisoned Patients with Amitraz Ingestion: Case Reports. Human Exp Toxicol. 2002;21(1):55-7.

18. Andrade SF, Sakate M. The Comparative Efficacy of Yohimbine and Atipamezole to Treat Amitraz Intoxication in Dogs. Vet Hum Toxicol. 2003;45(3):124-7.

19. Nichols M H, King W D, James L P. Clonidine Poisoning in Jefferson County, Alabama. Ann Emerg Med. 1997;29(4):511-7.

20. Schaffer D D, Hsu W H, Hopper D L. The Effects of Yohimbine and Four other Antagonists on Amitraz-induced Depression of Shuttle Avoidance Responses in Dogs. Toxicol Appl Pharmacol. 1990;104(3):543-7.

21. Holstege C P, Eldridge D L, Rowden A K. ECG Manifestations: The Poisoned Patient. Emerg Med Clin NAm. 2006;24:159-77.

22. Holstege C P, Dobmeier S G, Bechtel L K. Critical Care Toxicology. Emerg Med Clin N Am. 2008;25:715-39. 